\title{
Evaluation of healthy and sensory indexes of sweetened beverages using an electronic tongue
}

\author{
Luís G. Dias $^{\text {a }}$, Cédric Sequeira ${ }^{a}$, Ana C.A. Veloso ${ }^{\text {b,c }}$, Mara E.B.C. Sousa ${ }^{\text {a }}$, \\ António M. Peres ${ }^{\mathrm{d}, *}$ \\ a CIMO - Escola Superior Agrária, Instituto Politécnico de Bragança, Campus Santa Apolónia, Apartado 1172, 5301 -855 Bragança, Portugal \\ b Instituto Politécnico de Coimbra, ISEC, DEQB, Rua Pedro Nunes, Quinta da Nora, 3030-199 Coimbra, Portugal \\ ${ }^{\mathrm{C}}$ CEB - Centre of Biological Engineering, University of Minho, Campus de Gualtar, 4710-057 Braga, Portugal \\ d LSRE - Laboratory of Separation and Reaction Engineering - Associate Laboratory LSRE/LCM, Escola Superior Agrária, Instituto Politécnico de Bragança, \\ Campus Santa Apolónia, Apartado 1172, 5301-855 Bragança, Portugal
}

\section{H I G H L I G H T S}

- Overconsumption of soft-drinks and fruit-beverages may enhance health risks.

- Beverage's healthy and sensory indexes were calculated using chromatographic data.

- A potentiometric electronic tongue with multivariate linear models was applied.

- E-tongue discriminated samples according to glycemic load or fructose-intolerance levels.

- Healthy and sensory indexes were accurately quantified using E-tongue data.

\section{A R T I C L E I N F O}

\section{Article history:}

Received 16 June 2014

Received in revised form 28 July 2014

Accepted 5 August 2014

Available online 13 August 2014

\section{Keywords:}

Sugar sweetened beverages

Glycemic load

Fructose-intolerance index

Electronic tongue

Linear discriminant analysis

Simulated annealing algorithm

\section{GR A P H I C A L A B S T RAC T}
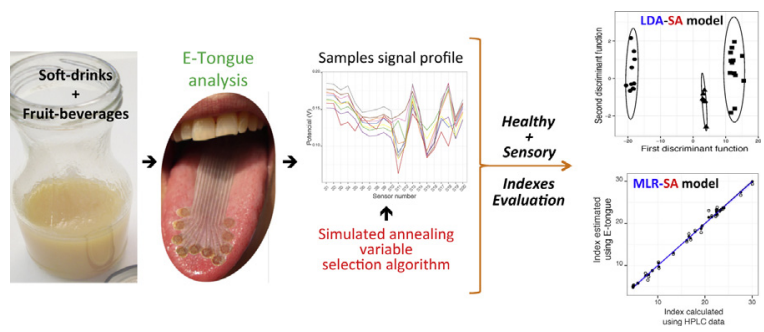

\begin{abstract}
A B S T R A C T
Overconsumption of sugar-sweetened beverages may increase the risk of health problems and so, the evaluation of their glycemic load and fructose-intolerance level is essential since it may allow establishing possible relations between physiologic effects of sugar-rich beverages and health. In this work, an electronic tongue was used to accurately classify beverages according to glycemic load (low, medium or high load) as well to their adequacy for people suffering from fructose malabsorption syndrome (tolerable or not): $100 \%$ of correct classifications (leave-one-out cross-validation) using linear discriminant models based on potentiomentric signals selected by a meta-heuristic simulated annealing algorithm. These results may be partially explained by the electronic tongue's capability to mimic the human sweetness perception and total acid flavor of beverages, which can be related with glycemic load and fructose-intolerance index. Finally, the E-tongue was also applied to quantify, accurately, healthy and sensory indexes using multiple linear regression models (leave-one-out cross-validation: $R_{\mathrm{adj}}>0.99$ ) in the following dynamic ranges: $4.7<$ glycemic load $\leq 30 ; 0.4<$ fructose intolerance index $\leq 1.5 ; 32<$ sweetness perception $<155$; $1.3<$ total acid flavor, $\mathrm{g} \mathrm{L}^{-1}<8.3$; and, $5.8<$ well-balanced flavor $\leq 74$. So, the proposed electronic tongue could be used as a practical, fast, low-cost and green tool for beverage's healthy and sensory evaluation. (C) 2014 Elsevier B.V. All rights reserved.
\end{abstract}

\footnotetext{
* Corresponding author. Tel.: +351 273303220; fax: +351 273325405.

E-mail addresses: Idias@ipb.pt (L.G. Dias), cedric.b.s@hotmail.com (C. Sequeira), anaveloso@isec.pt (A.C.A. Veloso), mebsousadias@gmail.com (M.E.B.C. Sousa), peres@ipb.pt (A.M. Peres).
} 


\section{Introduction}

The consumption of sugar-sweetened carbonated beverages (soft-drinks) and fruit beverages (e.g., nectars and juices) has experienced an increase since the 1980s. Soft-drinks, nectars and juices, among other requirements, must have a minimum percentage of added juice of $6-16 \%, 25-50 \%$ and equal to $100 \%$, respectively [1]. These non-alcoholic beverages are highly appreciated due to their sensory attributes. Human perception of beverage's specific flavors, balanced global flavor and sweetness are enhanced by sugars and organic acids contents, as well as by their equilibrium [2-5]. Besides, consumers also attributed to these beverages, especially to nectars and juices, healthy characteristics, being viewed as a source of essential nutrients (e.g., vitamins) and antioxidants. However, their overconsumption may increase the risk of health problems due to the high sugar content. Indeed, temporal studies have shown a close correlation between the upsurge in obesity and rising levels of the consumption of these beverages [6]. Also, diets rich in free or total fructose (considering its presence in other carbohydrates) can be highly prejudicial, inducing obesity, diabetes, dyslipidemia and insulin resistance [7]. This is of special relevance for people suffering from fructose intolerance, which may be partially prevented with the simultaneous ingestion of glucose [8], being envisaged a glucose/fructose ratio equal or greater than one.

So, from both consumer's and producer's point of view, it is of huge importance to evaluate healthy and sensory attributes by means of easily understandable indexes. Among the healthy indexes used in association studies of diet and chronic diseases [9] two are common: the glycemic load (GL), which quantifies the overall glycemic effect of a portion of food [7,9-12] and the fructose-intolerance (FI) index, mainly for people suffering from fructose malabsorption [8]. In fact, these indexes can be more useful nutritional concepts than the chemical classification of carbohydrates, since they may allow a better understanding of the relation between physiologic effects of carbohydrate-rich foods and health [10]. Among the sensory indexes the following are used as consumer's beverage acceptability or overall taste indicator: the total acid flavor, the sweetness index concept, used to assess fruit beverage sweetness as sucrose equivalent [13-15] and the wellbalanced flavor index, evaluated from the ratio between total sugars and total acids contents [2-4].

These indexes are influenced by the nature and source of carbohydrates and organic acids present in beverages and may be calculated from their contents, usually obtained experimentally by liquid chromatography based techniques [15-18]. These approaches, although accurate, are time-consuming, expensive, require qualified technical human resources and, usually, are not ambient friendly.

So, in the last years, fast, cost-effective and green electrochemical devices have been developed and applied, as alternative analytical techniques, for food matrices qualitative and/or quantitative analysis. The broad range of applicability of electronic tongues (E-tongue) within the food field, including direct analysis (e.g., milk, fruit-beverages, beer, wine) [19-22] or after sample pretreatment steps, such as dilution or extraction procedures (e.g., olive oils, honey and cereal-based solid foods) [23-27], may be attributed to the capability of these devices in recognizing and measuring basic taste compounds (e.g., acid, bitter, salty, sweet and umami) that has been previously demonstrated for multi-sensor systems based on lipid polymeric membranes $[22,28-30]$. In some cases, sample pre-treatment, like dilution, dissolution and/or extraction with a hydro-ethanolic solvent, is useful either to obtain a liquid sample, to minimize viscosity issues or to obtain a conductive solution, rich in tastant-related substances that can be recognized and measured by the E-tongue. Lipid/polymer membranes containing hydrophobic and hydrophilic groups, can be positively or negatively charged enabling the establishment of electrical interactions with electrolyte sourness tastants (e.g., organic acids) [31] or may allow electrostatic or hydrophobic interactions at the oil/water interface with sweet nonelectrolytes (e.g., sugars) [29,32].

Concerning non-alcoholic beverages evaluation, potentiometric E-tongues coupled with appropriate multivariate techniques have been successfully applied for discriminating different brands of apple juices [19] or orange juices [33-36]. Recently, a potentiometric E-tongue was used for semi-quantitative classification of fruit juices with different levels of added juice [37,38] and to quantify glucose and fructose contents in those beverages [38]. A potentiometric E-tongue was also applied as a sweetener recognition and taste predictor in coke soft-drinks [39]. More recently, an E-nose combined with a potentiometric E-tongue was reported for improving fruit juice recognition [40]. Most of these works report the classification of non-alcoholic beverages according to brand, beverage type or fruit flavor but, until now, none has focused on beverage classification according to healthy indexes.

In the present work, healthy and sensory indexes were calculated using experimental data regarding sugars and organic acids concentrations in beverages [18]. Based on GL or FI levels, beverages were split into 3 groups (low, medium or high GL) or 2 groups (FI index greater or lower than one, i.e., tolerable or not for people sensitive to fructose), independently of the beverage flavor, brand or commercial classification. The potential application of a potentiometric E-tongue to semi-quantitatively classify beverages according to healthy ratios (GL and FI index) was evaluated. Also the E-tongue's performance for quantifying both healthy and sensory indexes was investigated. For that, qualitative and quantitative chemometric tools were applied namely, linear discriminant analysis (LDA) and multiple linear regression (MLR) linear models, both coupled with a meta-heuristic simulated annealing variable selection algorithm. Also, possible linear correlations between healthy and sensory attributes were further assessed using the linear Pearson correlation coefficient (R-Pearson).

\section{Materials and methods}

\subsection{Materials}

\subsubsection{Samples}

Thirty commercial beverages samples ( 15 carbonated soft-drinks, 13 fruit-nectars and 2 fruit-juices) acquired in Bragança city (Portugal) were analyzed and their sugars (glucose, fructose and sucrose) and organic acids (ascorbic, citric and malic acids) contents were determined experimentally as previously reported [18]. Beverages were from different brands, several fruit flavors (e.g., orange, apple, pineapple, passion fruit, mango, red fruits and/or mixed fruits) and with added juice content ranging from 6 to $100 \%$ (according to label information).

\subsubsection{Reagents}

All the reagents used for HPLC analysis were of analytical grade and used as purchased: orthophosphoric acid, sucrose and ascorbic acid (Panreac); fructose, glucose, malic acid and acetic acid (Fluka); citric acid monohydrate (Fisher Scientific); and, tartaric acid (Riedel-deHaën). Regarding E-tongue, the reagents, from Fluka, were also of analytical grade and used as acquired: octadecylamine, oleyl alcohol, methyltrioctylammonium chloride and oleic acid as additives; bis(1-butylpentyl) adipate, dibutylsebacate, 2-nitrophenyl-octyl-ether, tris(2-ethylhexyl) 
phosphate, dioctyl phenylphosphonate as plasticizers; and, poly (vinyl chloride) polymer (PVC).

\subsection{Apparatus}

\subsubsection{HPLC equipment}

In this study, a HPLC Varian ProStar equipped with a 220 pump (Varian, Inc.), a 7725i Rheodyne manual injector, provided with a $20 \mu \mathrm{L}$ loop, a 7981 Jones Chromatography column oven, with a UV detector (Varian, model 9050) coupled to a RI detector (Varian, model RI-4), previously developed and in-house validated [18] was used to simultaneously separate and quantify the main organic acids (ascorbic, citric and malic acids, by UV at $210 \mathrm{~nm}$ ) and sugars (glucose, fructose and sucrose, by RI) contained in the beverage samples. The compounds separation was achieved using a thermostated $\left(45^{\circ} \mathrm{C}\right)$ Supelcogel $\mathrm{C}-610 \mathrm{H}$ size-exclusion column (SEC: C-610H model, $30 \mathrm{~cm} \times 7.8 \mathrm{~mm}$ id), using an isocratic elution, with a mobile phase free of organic solvents $(0.1 \%$ orthophosphoric acid aqueous solution).

\subsubsection{Electronic tongue device}

The E-tongue consisted of two print-screen potentiometric arrays, each one with 20 chemical sensors, with different crosssensitivity lipidic membranes (3\% of lipidic additive, $65 \%$ of plasticizer and $32 \%$ of PVC). In this study, a drop-by-drop technique was applied to prepare each membrane. Type of sensors and relative compositions were chosen based on a previous work [22] since, globally, they enable good signal stability in time (\%RSD $<5 \%)$ and repeatability $(0.5 \%<\% \mathrm{RSD}<15 \%)$ towards the basic standard taste compounds (e.g., sweet, acid, bitter, salty and umami). Further details on membrane composition of each sensor can be found in Sousa et al. [24]. Lipid/polymer membranes were used since they contain hydrophobic and hydrophilic groups allowing the interaction with several taste materials via electrostatic or hydrophobic interactions [41] being reported the existence of an electrical response in the presence of nonelectrolytes and electrolytes tastant substances [29-32], namely sugars and charged sweet compounds. A multi-sensor device was used since it is described that, in potentiometric principle, it could be difficult to evaluate sweetness with a single electrode, due to the nonelectrolyte or charged characteristics of sweet-responsive substances [30]. Each sensor was identified with a code with a letter $S$ (for sensor), followed by the number of the array ( 1 or 2 ), followed by the number of the membrane (1-20, corresponding to different combinations of plasticizer and additive used) [23,24]. So, the E-tongue comprised 20 sensors and their respective replicas which, although with the same composition, may have minor differences in the electrical response towards a specific sample, since inhomogeneous membranes may be obtained by the drop-by-drop technique used.

A multiplexer Agilent Data Acquisition Switch Unit model 349700A, controlled with the Agilent BenchLink Data Logger software installed on a PC, was used to record the potentiometric signals, that may vary from $-1.0 \mathrm{~V}$ to $+1.0 \mathrm{~V}$, from the 40 sensors setup (20 sensors in duplicate), which were measured against an $\mathrm{Ag} / \mathrm{AgCl}$ reference electrode (Crison, 5241). Samples were analyzed directly with the exception of soft-drinks, which needed to be degassed in an ultrasonic bath (Elma Transsonic 460/H, during $5 \mathrm{~min}$ ). To minimize potentiometric signals drift magnitude, all samples were evaluated in the same day in a maximum period time of $8 \mathrm{~h}$ (each analysis included a $10 \mathrm{~min}$ period for signal stabilization before recording the final signals profile followed by a new analysis after a cleaning step with deionized water and sample preparation, performed in 3-5 min). Duplicate sensors were used since it has been reported that the inclusion of repeated sensors in multivariate analysis can improve model performance [42] namely due to the slight variations of the membrane composition and physical properties (transparency and porosity) that may occur when a drop-by-drop technique is used for membrane preparation $[23,24]$.

\subsection{Procedures}

\subsubsection{Sugars and organic acids levels in beverage samples}

The main sugars and organic acids in the beverage samples studied were identified and determined using external calibration curves reported by Sequeira [18], using a HPLC in-house validated method, that reported the following contents: $10 \mathrm{~g} \mathrm{~L}^{-1}<$ glucose $<58 \mathrm{~g} \mathrm{~L}^{-1} ; \quad 13 \mathrm{~g} \mathrm{~L}^{-1}<$ fructose $<85 \mathrm{~g} \mathrm{~L}^{-1} ; \quad 0 \mathrm{~g} \mathrm{~L}^{-1} \leq$ sucrose $\leq 77 \mathrm{gL}^{-1} ; \quad 1 \mathrm{gL}^{-1}<$ citric acid $<9 \mathrm{gL}^{-1} ; 0 \mathrm{gL}^{-1}<$ malic acid $<5 \mathrm{gL}^{-1} ; 0 \mathrm{gL}^{-1}<$ ascorbic acid $<0.4 \mathrm{gL}^{-1}$. The chromatographic method had good linearity $(R>0.999)$, adequate detection and quantification limits $\left(0.020-0.091 \mathrm{~g} \mathrm{~L}^{-1}\right.$ and $0.060-0.28 \mathrm{~g} \mathrm{~L}^{-1}$, for sugars and organic acids, respectively), satisfactory instrumental precision (percentage relative standard deviation: $0.3 \%<\% \mathrm{RSD}$ $<3 \%$ and $0.2 \%<\% \mathrm{RSD}<6 \%$, for intra- and inter-days repeatabilities, respectively); acceptable repeatability and intermediate precision of the method $(0.3 \%<\% \mathrm{RSD}<2 \%$ and $0.2 \%<\% \mathrm{RSD}<5 \%$, respectively); and, acceptable method accuracy (percentage relative error: $\mathrm{RE} \%<5 \%)$

\subsubsection{Healthy indexes of beverages: calculation using HPLC data and beverages grouping}

Beverages samples were split according to the GLlevels or FI index (either free or available ratios). Concerning GL values, 3 groups were proposed: low (LGL: $\mathrm{GL}<11$ ), medium (MGL: $11 \leq \mathrm{GL}<20$ ) or high (HGL: GL $\geq 20$ ) glycemic load. Regarding the FI index and the envisaged value for minimizing fructose malabsorption, 2 groups were defined: beverages tolerable or not for people sensitive to fructose (TOL: FI index $>1$; NTOL: FI index $<1$, respectively). Healthy indexes were calculated using sugars composition data [18] for each beverage studied, according to:

(i) For GL data calculation a serving size (SS) of $330 \mathrm{~mL}$ was set (considering the mean SS values of beverages reported by Latulippe and Skoog [43]), and the carbohydrate composition data multiplied by the mean glycemic index (GI) of each pure sugar $\left(\mathrm{GI}_{\text {Glucose }}=99 \pm 7 ; \mathrm{GI}_{\text {Fructose }}=19 \pm 2 ; \mathrm{GI}_{\text {Sucrose }}=68 \pm 5\right)$ compiled by Foster-Powell et al. [10]:

$$
\begin{aligned}
& \mathrm{GI}_{\text {Sucrose }} \times\left[\text { Sucrose }, \mathrm{g} \mathrm{L}^{-1}\right]+\mathrm{GI}_{\text {Glucose }} \times\left[\text { Glucose }, \mathrm{g} \mathrm{L}^{-1}\right] \\
& \mathrm{GL}=\frac{+\mathrm{GI}_{\text {Fructose }} \times\left[\text { Fructose }, \mathrm{g} \mathrm{L}^{-1}\right]}{100} \\
& \times(\mathrm{SS}, \mathrm{L})
\end{aligned}
$$

(ii) The FI index was evaluated by the glucose-to-fructose free concentration ratio [8] and by the glucose-to-fructose total concentration ratio, assuming that each sucrose molecule may be transformed into one molecule of fructose and another of glucose [43]; since free fructose and fructose in sucrose may have different metabolic effects [44]:

$\mathrm{FI}_{\text {Free }}=\frac{\left[\text { Glucose, } \mathrm{g} \mathrm{L}^{-1}\right]}{\left[\text { Fructose }, \mathrm{g} \mathrm{L}^{-1}\right]}$

$\mathrm{FI}_{\text {Total }}=\frac{\left[\text { Glucose }, \mathrm{g} \mathrm{L}^{-1}\right]+\left[\text { Sucrose }, \mathrm{g} \mathrm{L}^{-1}\right] \times \mathrm{MW}_{\text {Glucose }} / \mathrm{MW}_{\text {Sucrose }}}{\left[\text { Fructose }, \mathrm{g} \mathrm{L}^{-1}\right]+\left[\text { Sucrose }, \mathrm{g} \mathrm{L}^{-1}\right] \times \mathrm{MW}_{\text {Fructose }} / \mathrm{MW}_{\text {Sucrose }}}$ 


\subsubsection{Sensory indexes of beverages: calculation using HPLC data}

For each beverage studied, sensory indexes were also calculated from the sugars and organic acids contents reported by Sequeira [18], with the aim of finding possible relations with the established groups defined based on the healthy indexes previously evaluated. The following sensory attributes were calculated:

(i) The sweetness perception (SWP) index of each beverage, an estimate of total human sweetness perception, was calculated as sucrose equivalent based on the amount and relative sweetness (RS) of each individual sugar $\left(\mathrm{RS}_{\text {Sucrose }}=1.00\right.$; $\left.\mathrm{RS}_{\text {Glucose }}=0.74 ; \mathrm{RS}_{\text {Fructose }}=1.73\right)[13-15]$ :

$\mathrm{SWP}=\mathrm{RS}_{\text {Sucrose }} \times\left[\right.$ Sucrose, $\left.\mathrm{g} \mathrm{L}^{-1}\right]+\mathrm{RS}_{\text {Glucose }}$ $\times\left[\right.$ Glucose, $\left.\mathrm{g} \mathrm{L}^{-1}\right]+\mathrm{RS}_{\text {Fructose }} \times$ [Fructose, $\mathrm{g} \mathrm{L}^{-1}$ ]

(ii) The total acid flavor $\left(\mathrm{TA}_{\text {Flavor }}\right)$ was calculated using the organic acids data composition [18] of each beverage:

$\mathrm{TA}_{\text {Flavor }}=\left[\right.$ Ascorbic acid, $\left.\mathrm{g} \mathrm{L}^{-1},\right]+\left[\right.$ Malic acid, $\left.\mathrm{g} \mathrm{L}^{-1}\right]$ $+\left[\right.$ Citric acid, $\left.\mathrm{g} \mathrm{L}^{-1}\right]$

(iii) The beverage well-balanced flavor $\left(\mathrm{WB}_{\text {Flavor }}\right)$ index, evaluated by the ratio between total sugars and total organic acids [2-4]:

$\mathrm{WB}_{\text {Flavor }}=\frac{\left[\text { Sucrose, } \mathrm{g} \mathrm{L}^{-1}\right]+\left[\text { Glucose, } \mathrm{g} \mathrm{L}^{-1}\right]+\left[\text { Fructose, } \mathrm{g} \mathrm{L}^{-1}\right]}{\mathrm{TA}_{\text {Flavor }}}$

\subsection{Statistical analysis}

Linear discriminant analysis (LDA) was applied, as a supervised learning technique, to classify the 30 beverage samples according to the groups emerging from each calculated healthy index (GL or FI index). A meta-heuristic variable selection technique, the simulated annealing (SA) algorithm, was applied to the E-tongue potentiometric sensor signals to select the most informative sensors subsets (i.e., independent variables), by eliminating redundant variables and increasing the accuracy of the classification model prediction. To minimize the risk of overoptimistic correct classification predictive performance, a leave-one-out cross-validation ( $\mathrm{LOO}-\mathrm{CV}$ ) procedure was used. This strategy has been recently applied with success by the research team $[23,24]$. So, SA algorithm was allowed to selected sub-sets containing between 2 and 29 sensors (which must be lower than the number of samples under study), being chosen the sub-set that enable the maximum correct predictive classification performance with the minimum number of sensors. Furthermore, bivariate correlations between calculated sensory data (sweetness perception index, well-balanced flavor index and total acid flavor) and the group centroids of each discriminant glycemic load cluster, obtained from the potentiometric E-tongue signals, were evaluated using the $R$-Pearson correlation coefficient, aiming to explain the potential of the E-tongue device in classifying beverages according to healthy indexes.

Finally, the use of multiple linear regression (MLR) models for quantifying both healthy (GL and FI levels) and sensory (sweetness perception index, well-balanced flavor index and total acid flavor) attributes of the studied beverages, based on the independent signals of the 40 potentiometric sensors contained in the E-tongue, was studied. Possible correlations among these indexes, since all of them are calculated using sugars and/or organic acids composition data, were also assessed using $R$-Pearson correlation coefficient. To obtain MLR models with the best predictive performances, sub-sets of 2-29 sensors (of the 40 available, assuming that sensor replicas can be treated as independent variables) were selected applying, once again, the SA algorithm. The most informative sub-set of sensors was select aiming to obtain a model with the minimum number of sensors and, an adjusted correlation coefficient $\left(R_{\text {adj }}\right)$ greater than 0.99 for the linear relation between indexes values estimated with E-tongue data versus indexes values calculated from experimental chromatographic data. To minimize the risk of overoptimistic results a LOO-CV procedure was also adopted. The acceptance of the E-tongue method as an alternative methodology for healthy and sensory indexes quantification was further evaluated. The predicted values, estimated by the MLR model using E-tongue signals, were compared with those calculated (Eqs. (1)-(6)) from the sugar composition data determined by HPLC, which was considered the reference procedure, by testing if the slope and intercept values for LOO-CV procedure were equal to the theoretical expected values (one and zero, respectively), from a statistical point of view [38].

All statistical data analysis was performed using the Subselect $[45,46]$ and MASS [47] packages of the open source statistical program R (version 2.15.1), at 5\% of significance level.

\section{Results and discussion}

\subsection{Sensory and healthy indexes calculation using sugars and organic acids HPLC profiles}

Healthy (GL and FI indexes) and sensory (SWP, TA Flavor $_{\text {and }}$ $\mathrm{WB}_{\text {Flavor }}$ indexes) attributes were calculated for each beverage sample (using the equations given in sub-Sections 2.3.2 and 2.3.3) and are shown in Table 1. Each calculated index showed values with high variability but, with no evident correlation with the sample's content of added juice $(R$-Pearson $<0.70)$.

Based on the calculated healthy indexes values, samples were grouped according to GL levels or FI ratios. For GL, 3 groups have been established (low, medium and high glycemic load) taking into account the GL values calculated for each beverage ( $\mathrm{GL}<11$; $11 \leq \mathrm{GL}<20$; and $\mathrm{GL} \geq 20$, respectively). Low medium and high GL groups contained 10, 7 and 13 samples, respectively. For the latter, beverages were split into 2 groups depending if FI index values were lower or greater than one $(\mathrm{NTOL}=24$ and $\mathrm{TOL}=6$ samples, respectively). All the supervised groups included samples of carbonated soft-drinks and fruit-beverages, from different brands and fruit flavors.

It should be noticed that GL values calculated in this work $(4 \leq \mathrm{GL} \leq 30)$ are in accordance with the mean GL values compiled by Foster-Powell et al. [10] for soft-drinks $(7 \leq \mathrm{GL} \leq 40)$ and fruit-beverages $(4 \leq \mathrm{GL} \leq 24)$.

Linear correlations between these indexes were evaluated through the calculation of $R$-Pearson coefficient. The results show that some of these indexes are linearly correlated ( $P$-values $<0.0001)$. A high positive linear correlation was obtained between SWP index and GL $(R$-Pearson $=0.92)$ demonstrating that sweeter beverages had greater GL values. On contrary, low linear correlations were found between $\mathrm{TA}_{\text {Flavor }}$ and $\mathrm{WB}_{\text {Flavor }}(R$-Pearson $=-0.71)$; $\mathrm{WB}_{\text {Flavor }}$ and GL $(R$-Pearson $=0.67)$; and $\mathrm{WB}_{\text {Flavor }}$ and SWP index $(R$-Pearson $=0.59)$. 
Table

Beverage samples analyzed: label information, calculated healthy and sensory indexes.

\begin{tabular}{|c|c|c|c|c|c|c|c|c|c|c|c|}
\hline \multirow[t]{2}{*}{ Sample no. } & \multirow[t]{2}{*}{ Main fruits in the composition } & \multicolumn{2}{|c|}{ Label information } & \multicolumn{5}{|c|}{ Healthy indexes calculated from HPLC data } & \multicolumn{3}{|c|}{ Sensory indexes calculated from HPLC data } \\
\hline & & $\overline{\text { Beverage type }^{a}}$ & Minimum juice \% & $\overline{\mathrm{GL}^{\mathrm{b}}}$ & GL group & $\mathrm{FI}_{\text {Free }}$ index $^{\mathrm{c}}$ & $\mathrm{FI}_{\text {Total }}$ index ${ }^{\mathrm{d}}$ & FI group & $\overline{\text { SWP index }}{ }^{\mathrm{e}}$ & $\mathrm{WB}_{\text {Flavor }}$ index $^{\mathrm{f}}$ & $\mathrm{TA}_{\text {Flavor }}$ index $\left(\mathrm{g} \mathrm{L}^{-1}\right)^{\mathrm{g}}$ \\
\hline 1 & Orange, mango & Nectar & 45 & 7.89 & LGL & 0.70 & 0.80 & NTOL & 50.4 & 8.03 & 5.16 \\
\hline 2 & Orange, apple, passion-fruit & Nectar & 50 & 7.38 & LGL & 0.51 & 0.60 & NTOL & 57.4 & 9.76 & 4.48 \\
\hline 3 & Orange & Nectar & 50 & 8.07 & LGL & 0.86 & 0.90 & NTOL & 48.5 & 6.17 & 6.63 \\
\hline 4 & Strawberry, apple & Nectar & 45 & 4.79 & LGL & 0.53 & 0.54 & NTOL & 41.8 & 5.83 & 5.21 \\
\hline 5 & Orange, carrot, mango & Nectar & 50 & 8.83 & LGL & 0.63 & 0.73 & NTOL & 59.8 & 8.80 & 5.48 \\
\hline 6 & Peach & Nectar & 50 & 23.4 & HGL & 1.10 & 1.03 & TOL & 115.2 & 26.9 & 4.02 \\
\hline 7 & Carrot, mango, tomato, apple, passion, kiwi, lemon & Soft-drink & 25 & 16.6 & MGL & 0.57 & 0.61 & NTOL & 132.7 & 39.2 & 2.55 \\
\hline 8 & Mango & Nectar & 30 & 21.4 & HGL & 0.74 & 0.88 & NTOL & 119.3 & 36.4 & 2.90 \\
\hline 9 & Apple & Juice & 100 & 17.0 & MGL & 0.43 & 0.48 & NTOL & 154.9 & 18.5 & 6.04 \\
\hline 10 & Red fruits & Nectar & 40 & 5.76 & LGL & 0.87 & 0.87 & NTOL & 39.2 & 7.33 & 4.22 \\
\hline 11 & Orange & Juice & 100 & 17.9 & MGL & 0.83 & 0.89 & NTOL & 105.8 & 10.9 & 8.22 \\
\hline 12 & Pineapple, coconut & Nectar & 43 & 10.1 & LGL & 1.01 & 1.01 & TOL & 57.3 & 13.8 & 3.60 \\
\hline 13 & Pear & Nectar & 50 & 16.1 & MGL & 0.44 & 0.71 & NTOL & 102.0 & 62.8 & 1.36 \\
\hline 14 & Grape and pomegranate fruits and green tea & Soft-drink & 20 & 4.73 & LGL & 0.87 & 0.87 & NTOL & 32.3 & 6.95 & 3.65 \\
\hline 15 & Orange, apple, pineapple, mango, apricot & Soft-drink & 20 & 22.4 & HGL & 0.87 & 0.94 & NTOL & 123.0 & 31.9 & 3.42 \\
\hline 16 & Pineapple, apple, orange, banana & Soft-drink & 20 & 24.0 & HGL & 0.88 & 0.96 & NTOL & 125.1 & 33.5 & 3.38 \\
\hline 17 & Apple, orange, pineapple, mango, guava, banana & Soft-drink & 20 & 22.4 & HGL & 0.84 & 0.90 & NTOL & 132.8 & 32.8 & 3.44 \\
\hline 18 & Strawberry & Soft-drink & 14 & 19.2 & MGL & 0.90 & 0.94 & NTOL & 111.0 & 38.9 & 2.45 \\
\hline 19 & Orange, pineapple, passion-fruit, apricot, guava, mango, banana & Soft-drink & 20 & 20.8 & HGL & 0.84 & 0.90 & NTOL & 121.3 & 27.5 & 3.79 \\
\hline 20 & Pineapple & Soft-drink & 20 & 23.1 & HGL & 0.88 & 0.95 & NTOL & 122.1 & 46.6 & 2.37 \\
\hline 21 & Orange & Soft-drink & 20 & 22.6 & HGL & 0.83 & 0.94 & NTOL & 118.9 & 27.5 & 3.92 \\
\hline 22 & Orange & Soft-drink & 10 & 19.4 & MGL & 1.40 & 1.23 & TOL & 96.6 & 46.5 & 1.90 \\
\hline 23 & Pineapple & Soft-drink & 8 & 22.3 & HGL & 1.18 & 1.11 & TOL & 117.6 & 74.0 & 1.42 \\
\hline 24 & Orange & Soft-drink & 8 & 22.6 & HGL & 1.50 & 1.32 & TOL & 111.4 & 50.5 & 2.02 \\
\hline 25 & Orange & Soft-drink & 11 & 10.2 & MGL & 0.88 & 0.94 & NTOL & 56.4 & 18.0 & 2.76 \\
\hline 26 & Pineapple & Soft-drink & 6 & 23.8 & HGL & 1.29 & 1.26 & TOL & 129.3 & 61.7 & 1.81 \\
\hline 27 & Tropical fruits & Soft-drink & 12 & 13.3 & MGL & 0.82 & 0.87 & NTOL & 82.6 & 18.0 & 3.81 \\
\hline 28 & Carrot, mango, tomato, apple, passion-fruit, kiwi, lemon & Nectar & 32 & 30.0 & HGL & 0.64 & 0.70 & NTOL & 217.5 & 43.2 & 3.91 \\
\hline 29 & Passion-fruit & Nectar & 25 & 27.6 & HGL & 0.90 & 0.96 & NTOL & 146.5 & 17.5 & 7.56 \\
\hline 30 & Strawberry, apple & Nectar & 45 & 4.90 & LGL & 0.58 & 0.61 & NTOL & 39.6 & 16.0 & 1.83 \\
\hline
\end{tabular}

LGL: low glycemic load, MGL: medium glycemic load, HGL: high glycemic load.

TOL and NTOL: tolerable or not for people sensitive to fructose.

${ }^{\text {a }}$ Beverage classification according to legal regulations [1].

b GL: glycemic load, calculated using Eq. (1).

$\mathrm{FF}_{\text {Free }}$ index: free fructose-intolerance index, calculated using Eq. (2).

e $\mathrm{FI}_{\text {Total }}$ index: total fructose-intolerance index, calculated using Eq. (3).

SWP index: sweetness perception index, calculated using Eq. (4).

WB Flavor index: well-balanced flavor index, calculated using Eq. (6).

${ }^{g}$ TA $A_{\text {Flavor: }}$ total acid flavor, calculated using Eq. (5). 


\subsection{E-tongue signals profiles of beverage samples}

The capability of the E-tongue to differentiate fruit-beverages with different healthy and sensory attributes, calculated using sugars and organic acids concentrations was evaluated. Regarding, sugars, this potential may be attributed to the different electrical response that is expected when potentiometric sensors, containing lipid/polymer membranes, are applied in aqueous solutions with different contents and chemical structures of sweet-tasting sugars (usually, monosaccharides show a lower electric response compared with disaccharides), although the underlying mechanism is not yet known [32]. Indeed, even if glucose, fructose and sucrose are nonelectrolytes, it has been suggested that the presence of two adjacent hydroxyl groups in a sugar molecule and the distance between them may lead to an electrical interaction between the surface of the lipid/polymeric membrane and the sugar molecules (for the same concentration, greater number of hydroxyl groups will generate a strongest electric response) [32]. This interaction would be similar to that described, based on studies concerning the structure-activity relationships, between hydroxyl groups in sweet-tasting sugars and the human sweet taste receptor [32]. In fact, literature $[29,32,48,49]$ reports that the electric response of potentiometric E-tongue to sugars may be due to the interaction on the surface of the lipid/polymer membrane (at the oil/water interface), either derived from the establishment of hydrogen bonds or due to the presence of some mediating electrolyte substances, between carboxyl or phosphate groups in the lipid/polymer membrane and the two vicinal hydroxyl groups of the sugar molecules. The response of the potentiometric E-tongue towards the organic acids present in the fruit beverages, may be explained since these molecules, which are electrolytes, electrically interact with the lipid/polymer membrane and after being adsorbed induce a change in the membrane potential [30,31].

The analysis of each beverage sample provided 40 potentiometric signals (20 different sensor membranes used in duplicate: $\mathrm{S} 1: 1-\mathrm{S} 1: 20$ and S2:1-S2:20). Fig. 1 shows the potentiometric signal's box plots for each sensor and each type of beverage studied (soft-drinks and fruit-beverages), for all the assays carried out. As can be seen from Fig. 1, sensors made with the same plasticizer and different additive compounds (e.g., S1:5-S1:8) have differences in signal intensities. This behavior could be attributed to the different functional groups of the additive compounds (amines, alcohol, acid and ammonium), leading to differences in the electrical or electrostatic interactions between the polymeric membrane and the target molecules (organic acids and sugars, respectively), resulting in different absorption ratios of each substance into the polymeric membrane. Similarly, differences between signals were observed for sensors made from different plasticizers (different functional groups) with the same additive (e.g., S1:1, S1:5, S1:9, $\mathrm{S} 1: 13$ and $\mathrm{S1:17)}$. Also, in general, when the same sensor was applied to different types of beverages, differences were observed in the signal intensities although the overall signal profile of the device showed a similar tendency. In few cases, comparable signals were recorded by the same sensor and different beverage types, which may be due to similar total sugar and/or organic acids contents of different types of beverages, resulting in an equivalent sensor response towards the global taste perception. Finally, as reported previously $[23,24]$, minor signal differences were also observed for sensor replicates. In fact, the device was homemade and the membrane formation accomplished using a drop-by-drop technique, where only temperature and time of drying were

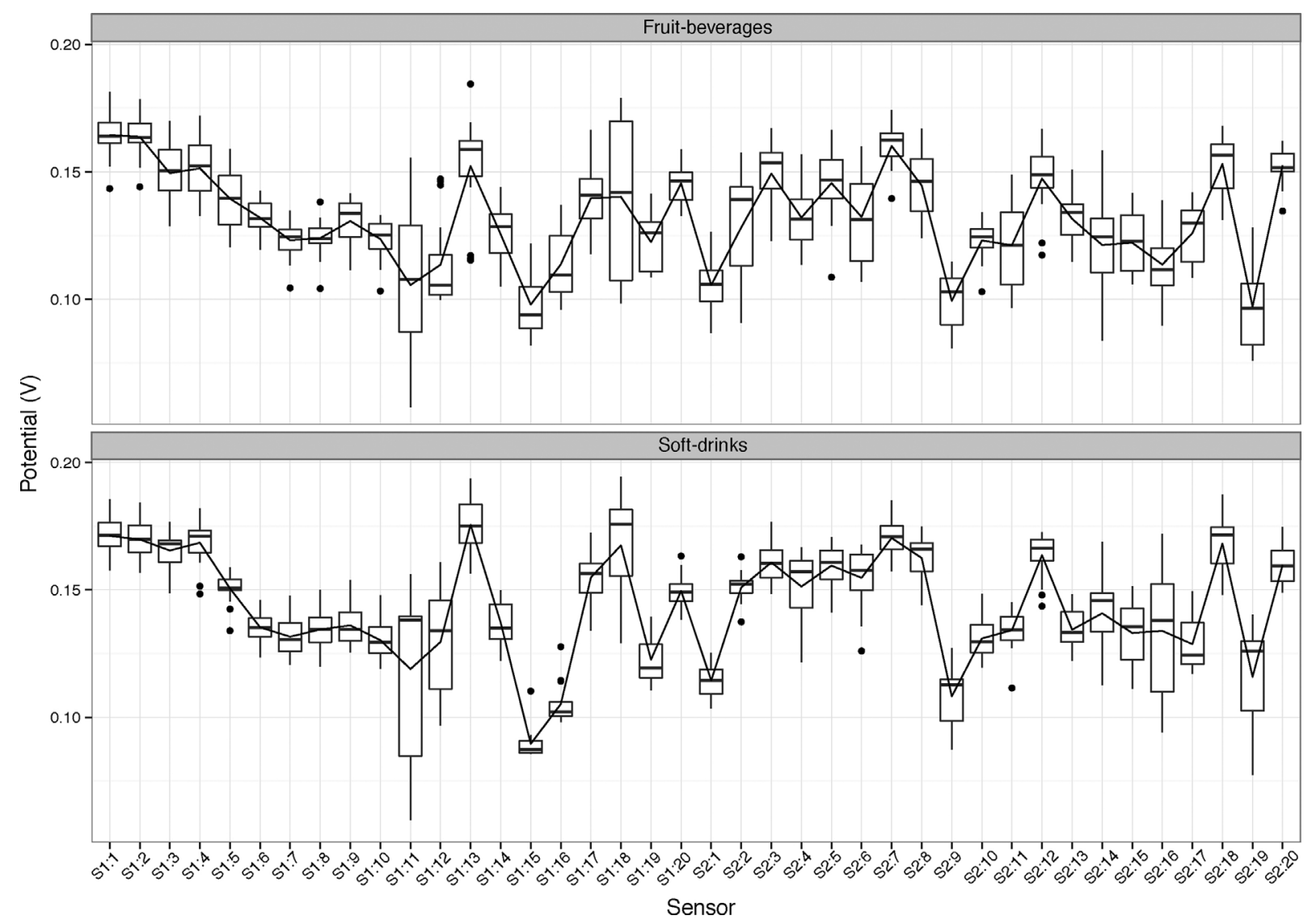

Fig. 1. Potentiometric E-tongue signal box-plots for the 15 soft-drinks and 15 fruit-beverages analysed. 
controlled, which could result in sensor duplicates with inhomogeneous thickness due to minor differences of solvent's evaporation rate and so with possible variations of the related physical properties (e.g., transparency and porosity). So, repeatability assays in time, were performed by analyzing a fruit-nectar sample, randomly selected (sample no. 3). The results showed satisfactory signal stability for all the sensors, when the sample was continuously analyzed during 3-h (maximum percentage variability between the initial and final recorded signals lower than $4.8 \%$, corresponding to a potential variation of $4 \mathrm{mV}$ ) as well as satisfactory signal repeatability when one beverage sample was analyzed 5 times in the same day (sensor's signal intra-day variability: $0.2 \%<\% \mathrm{RSD}<4.6 \%$ ) for all the sensors of the two arrays. Globally, signals intensities varied from $+0.06 \mathrm{~V}$ to $+0.20 \mathrm{~V}$ for all sensors included in the E-tongue, avoiding the need of data scaling. Due to the narrow range of signals intensities, a robust variable selection procedure should be implemented to select the most relevant signals sub-sets for establishing powerful predictive LDA models.

\subsection{E-tongue qualitative beverage's evaluation}

\subsubsection{Discrimination of beverages based on glycemic load levels}

The possibility of using the proposed electrochemical device to classify beverages with different GL levels was evaluated. For that, a LDA-SA procedure was applied to potentiometric signals recorded during beverages analysis with an E-tongue. A linear multivariate model, with 2 discriminant functions (explaining $100 \%$ of data variability), using a sub-set of 16 sensors (S1:1-S1:5, $\mathrm{S} 1: 8, \mathrm{~S} 1: 9, \mathrm{~S} 1: 12, \mathrm{~S} 1: 17, \mathrm{~S} 1: 19, \mathrm{~S} 2: 1, \mathrm{~S} 2: 4, \mathrm{~S} 2: 10, \mathrm{~S} 2: 13, \mathrm{~S} 2: 14$ and $\mathrm{S} 2: 17)$ was established. This sub-set of sensors indicates that replicas were used corroborating the findings reported by Correia et al. [42], which showed that including repeated sensors may improve the performance of multivariate models. This sensors sub-set also showed that all plasticizers and additives contributed to the E-tongue performance. Bis(1-butylpentyl) adipate and tris (2-ethyl-hexyl) phosphate plasticizers were the most and the less used, respectively, although based on the results of Toyota et al. [32], it was expected that the latter plasticizer had a higher influence since it contains a phosphate group, which is capable of producing a sweet-compound response. On the other hand, octadecylamine and oleyl alcohol were the additives that allowed obtaining a more precise fingerprint of each beverage showing a higher contribution for the E-tongue discrimination potential. This finding was expected since their related functional groups (amine and alcohol, respectively) may enhance the electrostatic interaction with sugar's vicinal hydroxyl groups [29]. The proposed model enabled $100 \%$ of correct classification prediction using LOO-CV procedure, as can be inferred by Fig. 2. The 1st discriminant function allows separating the 3 pre-defined GL groups, namely the LGL samples (negative quadrant) from MGL and HGL samples (positive quadrant). These results demonstrate the potential of the E-tongue plus LDA-SA as an effective methodology for discriminating and semi-quantitatively assess GL levels of beverages.

Also, $R$-Pearson correlation coefficients were evaluated trying to verify the existence of linear correlations between the spatial distribution of the mean group centroids (for the 1st discriminant function) of the GL groups (group centroids: $\mathrm{LGL}<\mathrm{MGL}<\mathrm{HGL}$ ) and the amount of added juice and the sensory indexes (SWP index, $\mathrm{WB}_{\text {Flavor }}$ ratio and $\mathrm{TA}_{\text {Flavor }}$ ). The results showed that no statistically significant correlation could be established with the amount of added juice $(R$-Pearson $=-0.58$ : $P$-value $=0.2223$ ), meaning that there is not a straightforward correlation between GL level and amount of added juice, and so this information, usually present in beverage's labels is not sufficient to infer about GL levels and their

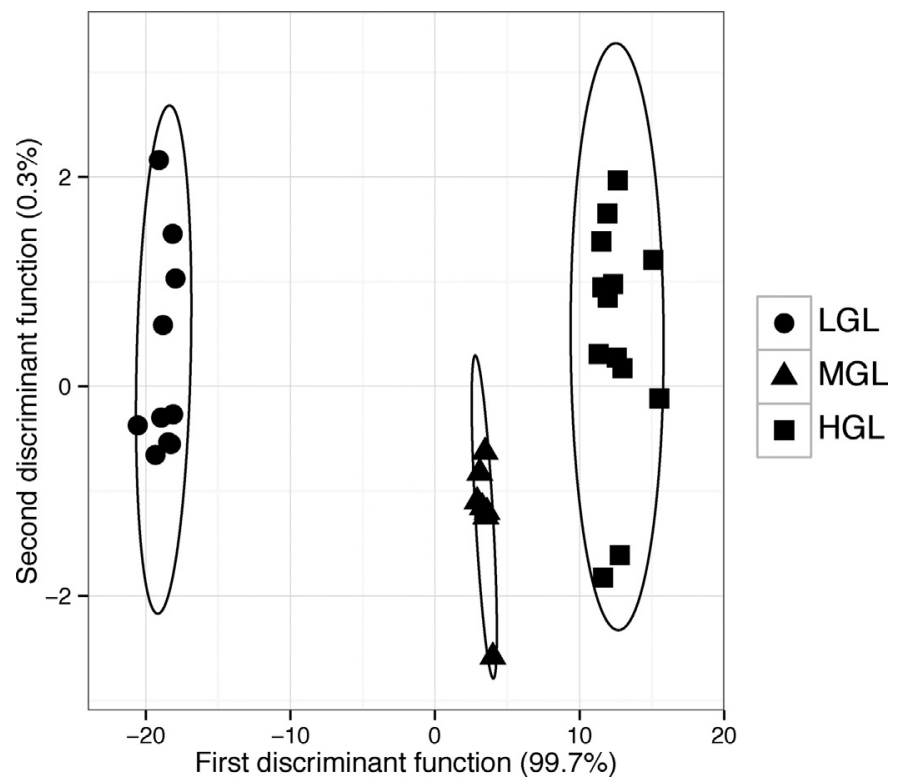

Fig. 2. Classification of beverages based on three semi-quantitative glycemic load levels using the two first LDA functions (explain 100\% of the total variance) based on the best E-tongue sensor signal sub-set (16 sensors) selected using the SA metaheuristic algorithm: low glycemic load (LGL: GL $<11$ ); medium glycemic load (MGL: $11 \leq \mathrm{GL}<20$ ); high glycemic load (HGL: $\mathrm{GL} \geq 20$ ).

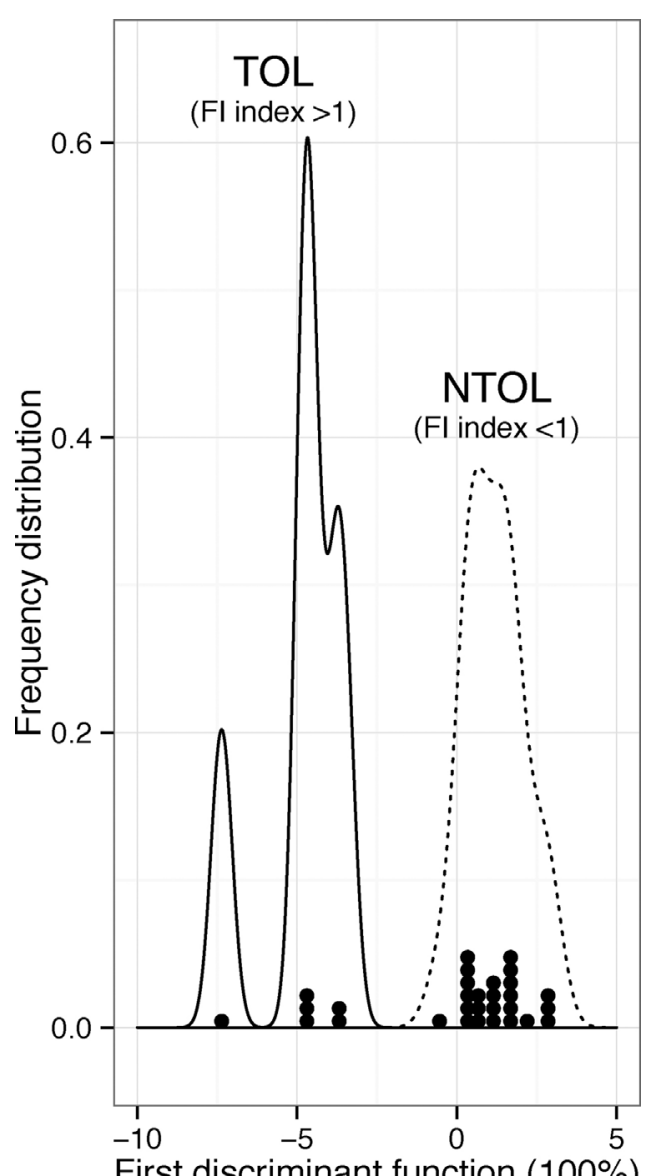

Fig. 3. Classification of beverages based on two fructose-intolerance index levels using the first LDA functions (explain 100\% of the total variance) based on the best E-tongue sensor signal sub-set (12 sensors) selected using the SA meta-heuristic algorithm: tolerable for people sensitive to fructose (TOL: FI index >1); not tolerable for people sensitive to fructose (NTOL: FI index $<1$ ). 
possible negative impact in consumer's health. On the contrary, significant correlations were found for beverages sensory attributes. A positive correlation was observed with beverage global sweetness, assessed using SWP index $(R$-Pearson $=0.9973$ : $P$-value $<0.0001)$ and $W_{B}$ Flavor ratio $(R$-Pearson $=0.9946: P$-value $<0.0001)$ showing that beverages with high GL levels are sweeter. A negative correlation $(R$-Pearson $=-0.9906$ : $P$-value $=0.0001)$ was noticed with the $\mathrm{TA}_{\text {Flavor }}$ index, showing that beverages with lower GL levels were more acid. Finally, these results also show that the good performance of the E-tongue, in classifying beverage samples according to their GL levels, may be mainly attributed to the ability of the device in mimic the human perception of two basic taste standards: sweet and acid.

3.3.2. Discrimination of beverages based on fructose-intolerance levels Beverages samples, independently of brand, amount of added juice or fruit flavor, were also split into 2 groups, depending if the FI index was greater (TOL group, 6 samples) or lower than one (NTOL group, 24 samples). This ratio may be used for classifying beverages as tolerable or not for people suffering from fructose malabsorption syndrome. A multivariate linear classification model was obtained, by applying a LDA-SA method to the 40 potentiometric signals recorded by the E-tongue device. A model with one linear discriminant function (which explained $100 \%$ of the data variability), based on the signals from 12 sensors (S1:4, S1:6, S1:8, S1:10, S1:12, S1:14, S1:17, S2:3, S2:12-S2:14 and S2:20), selected by applying the SA algorithm, was established allowing to classify beverages samples according to their FI index, namely if they could be classified as tolerable or not for people sensitive to fructose. The selected sensors sub-set demonstrated again that all plasticizers and additives were useful and could characterize properly the different beverages according to the different FI indexes. In this case, 2-nitrophenyl-octylether and tris (2-ethyl-hexyl) phosphate plasticizers coupled with oleyl alcohol and oleic acid additives seemed to be the combinations that enabled a more reliable differentiation of the studied beverages. The preferential use of phosphate based plasticizer is in accordance with the literature since it is reported that if the lipid/polymer membrane contains phosphate groups, its electrostatic interaction towards sugars is stronger [32]. The model enabled $100 \%$ of correct predictive classification using a LOO-CV procedure (Fig. 3). Again, it was demonstrated that the proposed E-tongue could be used as a practical tool for evaluating the FI index of soft-drinks and fruitbeverages, which could be easily implemented at industrial level. Again, this good performance may be attributed to the capability of the E-tongue in mimicking sweet and acid basic taste standards. Indeed, beverages with FI index higher than one (TOL group) are sweeter and with higher $\mathrm{WB}_{\text {Flavor }}$ values than beverages with FI index lower than one (NTOL group), containing the latter group higher levels of $\mathrm{TA}_{\text {Flavor }}$.

\subsection{E-tongue quantitative evaluation of health and sensory indexes of beverages}

Previously, Dias et al. [38] showed that potentiometric signal profiles of juices could be also used, together with appropriate

Table 2

Multiple linear regression parameters for LOO-CV procedure for healthy and sensory indexes.

\begin{tabular}{|c|c|c|c|c|}
\hline Healthy indexes $^{\mathrm{a}}$ & No. of sensors in MLR model & Model parameters & Leave-one-out cross-validation & $R_{\mathrm{adj}}$ \\
\hline GL & $19^{b}$ & $\begin{array}{l}\text { Slope } \\
\text { Slope } \mathrm{CI}^{\mathrm{h}} \\
\text { Intercept } \\
\text { Intercept } \mathrm{Cl}^{\mathrm{i}}\end{array}$ & $\begin{array}{l}0.994 \\
{[0.952,1.035]} \\
0.0765 \\
{[-0.682,0.836]}\end{array}$ & 0.9940 \\
\hline $\mathrm{FI}_{\text {Free }}$ & $21^{\mathrm{c}}$ & $\begin{array}{l}\text { Slope } \\
\text { Slope } \mathrm{CI}^{\mathrm{h}} \\
\text { Intercept } \\
\text { Intercept } \mathrm{CI}^{\mathrm{i}}\end{array}$ & $\begin{array}{l}1.010 \\
{[0.978,1.042]} \\
-0.00923 \\
{[-0.0372,0.0187]}\end{array}$ & 0.9966 \\
\hline $\mathrm{FI}_{\text {Total }}$ & $21^{\mathrm{d}}$ & $\begin{array}{l}\text { Slope } \\
\text { Slope } \mathrm{CI}^{\mathrm{h}} \\
\text { Intercept } \\
\text { Intercept } \mathrm{CI}^{\mathrm{i}}\end{array}$ & $\begin{array}{l}0.989 \\
{[0.950,1.028]} \\
0.00855 \\
{[-0.0266,0.0437]}\end{array}$ & 0.9946 \\
\hline Sensory indexes ${ }^{a}$ & No. of sensors in MLR model & Model parameters & Leave-one-out cross-validation & $R_{\mathrm{adj}}$ \\
\hline SWP & $21^{\mathrm{e}}$ & $\begin{array}{l}\text { Slope } \\
\text { Slope } \mathrm{CI}^{\mathrm{h}} \\
\text { Intercept } \\
\text { Intercept } \mathrm{CI}^{\mathrm{i}}\end{array}$ & $\begin{array}{l}0.994 \\
{[0.971,1.017]} \\
0.417 \\
{[-2.054,2.887]}\end{array}$ & 0.9982 \\
\hline $\mathrm{WB}_{\text {Flavor }}$ & $22^{f}$ & $\begin{array}{l}\text { Slope } \\
\text { Slope } \mathrm{CI}^{\mathrm{h}} \\
\text { Intercept } \\
\text { Intercept } \mathrm{CI}^{\mathrm{i}}\end{array}$ & $\begin{array}{l}0.990 \\
{[0.956,1.023]} \\
0.304 \\
{[-0.831,1.439]}\end{array}$ & 0.9960 \\
\hline $\mathrm{TA}_{\text {Flavor }}$ & $19^{\mathrm{g}}$ & $\begin{array}{l}\text { Slope } \\
\text { Slope } \mathrm{CI}^{\mathrm{h}} \\
\text { Intercept } \\
\text { Intercept } \mathrm{CI}^{\mathrm{i}}\end{array}$ & $\begin{array}{l}0.982 \\
{[0.934,1.030]} \\
0.0808 \\
{[-0.119,0.281]}\end{array}$ & 0.9918 \\
\hline
\end{tabular}

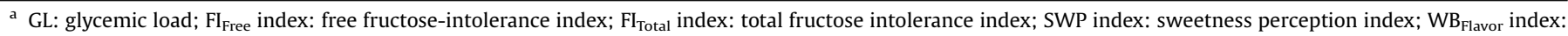
well-balanced flavor index; $\mathrm{TA}_{\mathrm{Flavor}}$ : total acid flavor.

b S1:3, S1:4, S1:8, S1:9, S1:13, S1:16-S1:19, S2:1, S2:3, S2:4, S2:6, S2:8, S2:9, S2:13-S2:15, S2:18.

c $\mathrm{S} 1: 1, \mathrm{~S} 1: 3, \mathrm{~S} 1: 5, \mathrm{~S} 1: 6, \mathrm{~S} 1: 12, \mathrm{~S} 1: 13, \mathrm{~S} 1: 15, \mathrm{~S} 1: 17-\mathrm{S} 1: 19, \mathrm{~S} 2: 1, \mathrm{~S} 2: 4-\mathrm{S} 2: 6, \mathrm{~S} 2: 9-\mathrm{S} 2: 11, \mathrm{~S} 2: 13, \mathrm{~S} 2: 16, \mathrm{~S} 2: 18, \mathrm{~S} 2: 19$.

d $\mathrm{S} 1: 1, \mathrm{~S} 1: 3-\mathrm{S} 1: 8, \mathrm{~S} 1: 10-\mathrm{S} 1: 12, \mathrm{~S} 1: 14, \mathrm{~S} 1: 16, \mathrm{~S} 2: 2, \mathrm{~S} 2: 4, \mathrm{~S} 2: 6-\mathrm{S} 2: 8, \mathrm{~S} 2: 10, \mathrm{~S} 2: 14, \mathrm{~S} 2: 17, \mathrm{~S} 2: 19$.

e $\mathrm{S} 1: 2, \mathrm{~S} 1: 3, \mathrm{~S} 1: 4, \mathrm{~S} 1: 6, \mathrm{~S} 1: 8, \mathrm{~S} 1: 9, \mathrm{~S} 1: 14, \mathrm{~S} 1: 15, \mathrm{~S} 1: 17, \mathrm{~S} 1: 18, \mathrm{~S} 2: 1, \mathrm{~S} 2: 2-\mathrm{S} 2: 4, \mathrm{~S} 2: 6, \mathrm{~S} 2: 8, \mathrm{~S} 2: 9, \mathrm{~S} 2: 13-\mathrm{S} 2: 15, \mathrm{~S} 2: 20$.

f $\mathrm{S} 1: 1, \mathrm{~S} 1: 2, \mathrm{~S} 1: 6, \mathrm{~S} 1: 8-\mathrm{S} 1: 11, \mathrm{~S} 1: 16-\mathrm{S} 1: 20, \mathrm{~S} 2: 1, \mathrm{~S} 2: 6, \mathrm{~S} 2: 7, \mathrm{~S} 2: 12-\mathrm{S} 2: 17, \mathrm{~S} 2: 19$.

g S1:3, S1:5, S1:11, S1:13-S1:18, S1:20, S2:1, S2:6-S2:9, S2:14, S2:16, S2:17, S2:19.

h Slope CI: slope's 95\% confidence interval.

${ }^{\mathrm{i}}$ Intercept CI: intercept's 95\% confidence interval. 
multivariate linear models, to estimate glucose and fructose concentrations, suggesting that E-tongues could have a wider set of applications. In this work, for the first time, signals profiles of an E-tongue were used to establish MLR models for evaluating healthy (GL and FI index) and sensory (SWP, WB $\mathrm{W}_{\text {Flavor }}$ and $\mathrm{TA}_{\text {Flavor }}$ indexes) of soft-drinks and fruit-beverages.

MLR models were established for each calculated index using the potentiometric signals recorded by the E-tongue. Sub-sets of sensors, from 2 to 29 signals of the 40 sensors available (20 different sensors and replicas), with a suitable predictive ability, were selected aiming to obtain a LOO-CV adjusted correlation coefficient $\left(R_{\mathrm{adj}}\right)$ higher than 0.99 and the lowest number of sensors (i.e., independent variables). The regression results for the LOO-CV for each index are listed in Table 2. The MLR models established had a satisfactory fitting predictive performance, with LOO-CV adjusted correlation coefficients higher than 0.99, allowing explaining at least $98 \%$ of data variability. The slope and intercept values, as well as the respective 95\% confidence intervals, support the good predictive accuracy of the MLR models. Indeed, from the previous results it is possible to conclude that there is no statistical evidence, at $5 \%$ of significance level, that the slope and intercept values of each regression line are different from theoretical expected values (slope equal to one; intercept equal to zero). This conclusion may lead to the acceptance of E-tongue plus LDA-SA as a possible quantification procedure for healthy and sensory indexes evaluation in soft-drinks and fruit-beverages. The overall, satisfactory results are gathered in Fig. 4. This figure shows the comparison between healthy or sensory index calculated by the MLR models, established using the E-tongue data, and the indexes
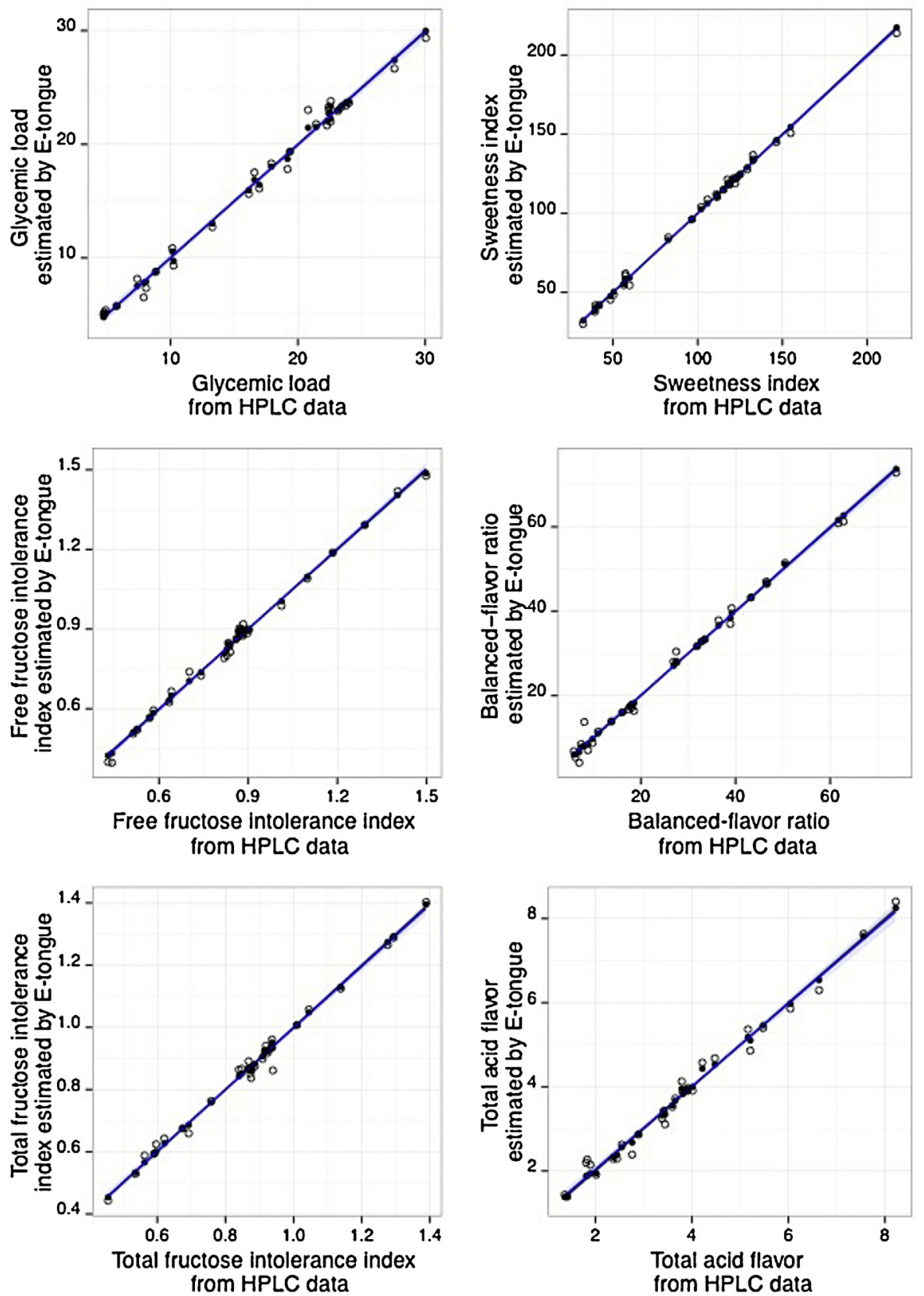

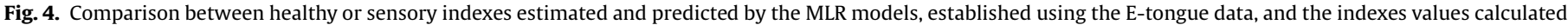

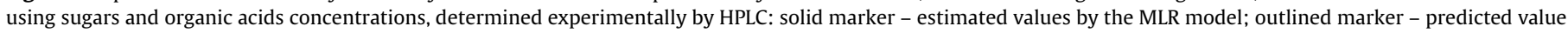
obtained by the LOO-CV procedure. 
values calculated using sugars and organic acids concentrations, determined experimentally by HPLC.

\section{Conclusions}

In this work it was demonstrated, for the first time, that a potentiometric E-tongue coupled with a LDA-SA procedure could be used as a practical tool to successfully classify carbonated softdrinks and fruit beverages, from different brands, flavor and types, according to different levels of glycemic load or fructoseintolerance index. Moreover, the E-tongue based methodology was able to accurately quantify beverage's healthy and sensory indexes. So, the proposed electrochemical methodology may be used as a practical alternative analytical tool reducing the use of time-consuming and more expensive analytical techniques. These good performances of the electronic tongue may potentiate the development of electrochemical devices and future application within food healthy indexes evaluation, namely those that may be correlated to taste indicators. Nevertheless, it should be remarked, that the mechanism of electrostatic interactions between the lipid/polymer membranes and the sugar target molecules is not fully understood, implying the need of further studies.

\section{Acknowledgements}

This work was partially co-financed by FCT and FEDER under Program COMPETE (Project PEst-C/EQB/LA0020/2013); by the Strategic Project PEst-OE/EQB/LA0023/2013 and by the project ref. RECI/BBB-EBI/0179/2012 (project number FCOMP01-0124-FEDER-027462) funded by Fundação para a Ciência e a Tecnologia.

\section{References}

[1] P.R. Ashurst, Chemistry and Technology of Soft Drinks and Fruit Juices, second ed., Blackwell Publishing, Hereford, United Kingdom, 2005.

[2] L.A. Terry, S.F. White, L.A. Tigwell, The application of biosensors to fresh produce and the wider food industry, J. Agr. Food Chem. 53 (2005) 1309-1319.

[3] A. Keutgen, E. Pawelzik, Modifications of taste-relevant compounds in strawberry fruit under $\mathrm{NaCl}$ salinity, Food Chem. 105 (2007) 1487-1494.

[4] J.G. Bordonaba, L.A. Terry, Biochemical profiling and chemometric analysis of seventeen UK-grown black currant cultivars, J. Agr. Food Chem. 56 (2008) 7422-7430.

[5] P. Crespo, J.G. Bordonaba, L.A. Terry, C. Carlen, Characterisation of major taste and health-related compounds of four strawberry genotypes grown at different Swiss production sites, Food Chem. 122 (2010) 16-24.

[6] F.B. Hu, V.S. Malik, Sugar-sweetened beverages and risk of obesity and type 2 diabetes: epidemiologic evidence, Physiol. Behav. 100 (2010) 47-54.

[7] T.P. Trinidad, A.C. Mallillin, R.S. Sagum, R.R. Encabo, Glycemic index of commonly consumed carbohydrate foods in the Philippines, J. Funct. Foods 2 (2010) 271-274.

[8] J.E. Riby, T. Fujisawa, N. Kretchmer, Fructose absorption, Am. J. Clin. Nutr. 58 (1993) 748S-753S.

[9] C.L. Martin, S.P. Murphy, D.L.M. Au, Compiling glycemic index and glycemic load values for addition to a food composition database, J. Food Comp. Anal. 2 (2008) 469-473.

[10] K. Foster-Powell, S.H.A. Holt, J.C. Brand-Miller, International table of glycemic index and glycemic load values: 2002, Am. J. Clin. Nutr. 76 (2002) 5-56.

[11] M. Rossi, L. Lipworth, J. Polesel, E. Negri, C. Bosetti, R. Talamini, J.K. McLaughlin, C. La Vecchia, Dietary glycemic index and glycemic load and risk of pancreatic cancer: a case-control study, Ann. Epidemiol. 20 (2010) 460-465.

[12] M.H. Rouhani, A. Salehi-Abargouei, L. Azadbakht, Effect of glycemic index and glycemic load on energy intake in children, Nutrition 29 (2013) 1100-1105.

[13] E.A. Baldwin, J.W. Scott, M.A. Einstein, T.M.M. Malundo, B.T. Carr, R.L. Shewfelt K.S. Tandon, Relationship between sensory and instrumental analysis for tomato flavor, J. Am. Soc. Hortic. Sci. 123 (1998) 906-915.

[14] J.M. Obando-Ulloa, I. Eduardo, A.J. Monforte, J.P. Fernández-Trujillo, Identification of QTLs related to sugar and organic acid composition in melon using near-isogenic lines, Sci. Hortic. 121 (2009) 425-433.

[15] T. Mahmood, F. Anwar, M. Abbas, M.C. Boyce, N. Saari, Compositional variation in sugars and organic acids at different maturity stages in selected small fruits from Pakistan, Int. J. Mol. Sci. 13 (2012) 1380-1392.

[16] P. Muñoz-Robredo, P. Robledo, D. Manríquez, R. Molina, B.G. Defilippi, Characterization of sugars and organic acids in commercial varieties of table grapes, Chil. J. Agr. Res. 71 (2011) 452-458.
[17] H.A. Eyéghé-Bickong, E.O. Alexandersson, L.M. Gouws, P.R. Young, M.A. Vivier, Optimisation of an HPLC method for the simultaneous quantification of the major sugars and organic acids in grapevine berries, J. Chromatogr. B 885-886 (2012) 43-49.

[18] C.B. Sequeira, Análise de açúcares e ácidos orgânicos em sumos comerciais: aplicação de HPLC-SEC-UV-IR e língua eletrónica, Master Thesis in Food Quality and Safety, Polytechnic Institute of Bragança, Bragança, Portugal, 2012.

[19] P. Ciosek, Z. Brzózka, W. Wróblewski, Classification of beverages using a reduced sensor array, Sens. Actuators B Chem. 103 (2004) 76-83.

[20] A. Legin, A. Rudnitskaya, L. Lvova, Y. Vlasov, C. Di Natale, A. D’Amico, Evaluation of Italian wine by electronic tongue: recognition, quantitative analysis and correlation with human sensory perception, Anal. Chim. Acta 484 (2003) 3344.

[21] P. Ciosek, W. Wróblewski, The recognition of beer with flow-through sensor array based on miniaturized solid-state electrodes, Talanta 69 (2006) 11561161.

[22] L.G. Dias, A.M. Peres, A.C.A. Veloso, F.S. Reis, M. Vilas Boas, A.A.S.C. Machado, An electronic tongue taste evaluation: identification goat milk adulterations with bovine milk, Sens. Actuators B Chem. 136 (2009) 209-217.

[23] L.G. Dias, A. Fernandes, A.C.A. Veloso, A.A.S.C. Machado, J.A. Pereira, A.M. Peres, Single-cultivar extra virgin olive oil classification using a potentiometric electronic tongue, Food Chem. 160 (2014) 321-329.

[24] M.E.B.C. Sousa, L.G. Dias, A.C.A. Veloso, L. Estevinho, A.M. Peres, A.A.S.C. Machado, Practical procedure for discriminating monofloral honeys with a broad pollen profile variability using an electronic tongue, Talanta 128 (2014) 284-292.

[25] A.M. Peres, L.G. Dias, A.C.A. Veloso, S.G. Meirinho, J. Sá Morais, A.A.S.C. Machado, An electronic tongue for gliadins semi-quantitative detection in foodstuffs, Talanta 83 (2011) 857-864.

[26] I. Escriche, M. Kadar, E. Domenech, L. Gil-Sánchez, A potentiometric electronic tongue for the discrimination of honey according to the botanical origin. Comparison with traditional methodologies: physicochemical parameters and volatile profile, J. Food Eng. 109 (2012) 449-456.

[27] N. Major, K. Marković, M. Krpan, G. Šarić, M. Hruškar, N. Vahčić, Rapid honey characterization and botanical classification by an electronic tongue, Talanta 85 (2011) 569-574

[28] M. Habara, D. Beppu, H. Cui, H. Ikeazaki, K. Toko, Detection of sugars using lipid/polymer membranes, Sens. Mater. 19 (2007) 325-331.

[29] K. Toyota, H. Cui, K. Abe, M. Habara, K. Toko, H. Ikeazaki, Sweetness sensor with lipid/polymer membranes: sweet-responsive susbtances, Sens. Mater. 23 (2011) 465-474.

[30] M. Yasuura, H. Okazaki, Y. Tahara, H. Ikezaki, K. Toko, Development of sweetness sensor with selectivity to negatively charged high-potency sweeteners, Sens. Actuators B 201 (2014) 329-335.

[31] M. Yasuura, Y. Tahara, H. Ikezaki, K. Toko, Development of a sweetness sensor for aspartame, a positively charged high-potency sweetener, Sensors 14 (2014) 7359-7373.

[32] K. Toyota, H. Cui, K. Abe, M. Habara, K. Toko, H. Ikeazaki, Sweetness sensor with lipid/polymer membranes: response to various sugars, Sens. Mater. 23 (2011) 475-482.

[33] P. Ciosek, Z. Brzózka, W. Wróblewski, Electronic tongue for flow-through analysis of beverages, Sens. Actuators B Chem. 118 (2006) 454-460.

[34] P. Ciosek, R. Mamińska, A. Dybko, W. Wróblewski, Potentiometric electronic tongue based on integrated array of microelectrodes, Sens. Actuators B Chem. 127 (2007) 8-14.

[35] P. Ciosek, W. Wróblewski, Performance of selective and partially selective sensors in the recognition of beverages, Talanta 71 (2007) 738-746.

[36] M. Liu, J. Wang, D. Li, M. Wang, Electronic tongue coupled with physicochemical analysis for the recognition of orange beverages, J. Food Quality 35 (2012) 429-441.

[37] A.M. Peres, L.G. Dias, T.P. Barcelos, J. Sá Morais, A.A.S.C. Machado, An electronic tongue for juice level evaluation in non-alcoholic beverages, Proceedings of the Eurosensors XXIII conference, Procedia Chem. 1 (2009) 1023-1026.

[38] L.G. Dias, A.M. Peres, T.P. Barcelos, J. Sá Morais, A.A.S.C. Machado, Semiquantitative and quantitative analysis of soft drinks using an electronic tongue, Sens. Actuators B Chem. 154 (2011) 111-118.

[39] D. Szöllösi, Z. Kovács, A. Gere, L. Sipos, Z. Kókai, A. Fekete, Sweetener recognition and taste prediction of coke drinks by electronic tongue, IEEE Sens. J. 12 (2012) 3119-3123.

[40] Z. Haddi, S. Mabrouk, M. Bougrini, K. Tahri, K. Sghaier, H. Barhoumi, N. El Bari, A Maaref, N. Jaffreziz-Renault, B. Bouchikhi, E-nose and E-tongue combination for improved recognition of fruit juice samples, Food Chem. 150 (2014) 246-253.

[41] Y. Kobayashi, M. Habara, H. Ikezazki, R. Chen, Y Naito, K. Toko, Advanced taste sensors based on artificial lipids with global selectivity to basic taste qualities and high correlation to sensory scores, Sensors 10 (2010) 3411-3443.

[42] D.P.A. Correia, J.M.C.S. Magalhães, A.A.S.C. Machado, Array of potentiometric sensors for simultaneous analysis of urea and potassium. Talanta 67 (2005) 773-782.

[43] M.E. Latulippe, S.M. Skoog, Fructose malabsorption and intolerance: effects of fructose with and without simultaneous glucose ingestion, Crit. Rev. Food Sci. Nutr. 51 (2011) 583-592.

[44] EFSA Panel on Dietetic Products, Nutrition and Allergies (NDA), Guidance on the scientific requirements for health claims related to bone, joints, skin and oral health, EFSA J. 10 (5) (2012) 2702 [13pp]. doi:10.2903/j.efsa.2012.2702 (www.efsa.europa.eu/efsajournal). 
[45] J. Cadima, J.O. Cerdeira, M. Minhoto, Computational aspects of algorithms for variable selection in the context of principal components, Comput. Stat. Data Anal. 47 (2004) 225-236.

[46] J. Cadima, J.O. Cerdeira, P.D. Silva, M. Minhoto, The Subselect R package. (http:// cran.r-project.org/web/packages/subselect/vignettes/subselect.pdf), 2012.

[47] W.N. Venables, B.D. Ripley, Modern Applied Statistics with S, fourth ed., Springer, New York, 2002.
[48] S. Ishizaka, S. Kinoshita, Y. Nishijima, N. Kitamura, Direct observation of molecular recognition mediated by triple hydrogen bonds at a water/oil interface: time-resolved total internal reflection fluorometry study, Anal. Chem. 75 (2003) 6035-6042.

[49] S. Nishizawa, T. Yokobori, R. Kato, K. Yoshimoto, T. Kamaishi, N. Teramae, Hydrogen-bond forming ionophore for highly efficient transport of phosphate anions across the nitrobenzene-water interface, Analyst 128 (2003) 663-669. 